\title{
Availability of facilities and equipment to provide emergency care in ambulances, which transport patients to the National Hospital of Sri Lanka
}

\author{
Gangadevi Nandasena ${ }^{1}$ \& Chrishantha Abeysena ${ }^{2 *}$ \\ ${ }^{1}$ Ministry of Health, Sri Lanka; ${ }^{2}$ Department of Public Health, Faculty of Medicine, University of Kelaniya, Sri Lanka \\ *Correspondence: chrishanthaabeysena@yahoo.com
}

DOI: https://doi.org/10.4038/jccpsl.v24i4.8154

Received on: 20 April 2018

Accepted on: 03 October 2018

\begin{abstract}
Background: Ambulance is a specially designed, well-equipped vehicle used to transport patients securely until they are handed over to the place of definitive care. The aim of ambulance services is to save the life of a person before reaching hospital.

Objective: To describe available facilities and equipment to provide essential emergency care in ambulances that transport patients to the National Hospital of Sri Lanka

Methods: A hospital-based, cross-sectional study was carried out at the National Hospital of Sri Lanka from August to October 2008. All ambulances that arrived at the hospital during the study period with an emergency patient were selected for the study $(n=409)$. A checklist was used to assess in-situ facilities and equipment that should be available for basic life support (BLS) and advanced life support (ALS).

Results: Most of the ambulances were equipped only with very basic facilities such as wheeled stretcher $95.4 \%$ $(n=390)$, fire extinguishers $75.3 \%(n=308)$ and warning siren $94.4 \%(n=386)$. Among the ventilation and air way equipment, oxygen cylinders showed the highest $(n=281,68.7 \%)$ availability in ambulances while oro-pharyngeal air ways were available only in $4.9 \%(n=20)$. Ventilation and air way equipment required for ALS were available only in less than $1 \%$ of the ambulances. Among patient assessment equipment, pen lights were available in $19.6 \%$ $(n=80)$ and blood pressure apparatus in 2.2\% $(n=9)$. Obstetrical supplies, splinting equipment, surgical dressings except bandages, drug delivery devices and intravenous equipment except intravenous hook were available in less than 5\% of all ambulances. Most of the ambulances were equipped with safety and accessory equipment except child safety seat $(n=2,0.5 \%)$, triage tags $(n=7,1.7 \%)$ and hack saw $(n=3,0.7 \%)$, which were available in less than $2 \%$ of all ambulances.
\end{abstract}

Conclusions: The facilities and equipment that should be available in both BLS and ALS ambulances were poor.

Key words: ambulance, emergency care, facilities, life support, quality 


\section{Introduction}

Ambulance is a specially designed, well-equipped vehicle used to transport patients securely until they are handed over to the place of definitive care. Universally, ambulances are used for two types of patient transport: transport of patients in an emergency from a scene to hospital or place of definitive care (emergency medical services) and inter-hospital transport of patients from lower level of care to a facility of higher level (retrieval medical service).

Currently, ambulance services have developed throughout the world to an extent that they can provide all facilities and services available in intensive care units at the roadside or within an aircraft (air ambulances). The aim of ambulance services is to save the life of a person before reaching hospital (pre-hospital setting). Therefore, it should be equipped with at least minimum life-saving and rescue equipment. These facilities and equipment range from extremely basic material such as simple dressing material (bandages and gauze) to advanced equipment such as cardiac defibrillators and ventilators. According to the availability of facilities, there are two types of ambulances: basic life support (BLS) and advanced life support (ALS) ambulances (1). The BLS ambulances have minimum facilities in providing basic resuscitation for patients, whereas the ALS ambulances are equipped with more advanced equipment to provide advanced treatment for patients. The American College of Surgeons developed a standard list of equipment for ambulances (2). A study done in Lithuania showed that the ambulances lack some important equipment, which provide first medical aid in trauma cases (3).

Government ambulance services in Sri Lanka first started in 1947 with 13 ambulances, which have been strengthened now, with nearly 800 ambulances islandwide. Sri Lankan ambulance services are mainly hospital-based and are aimed at transporting patients. In Sri Lanka, pre-hospital care or emergency medicine is not yet developed as a medical sub-specialty. The ambulance services function as a method of patient transport and retrieval service rather than as an emergency medical service. Very few communitybased ambulance services are available in Sri Lanka. Though most of the public health indicators are available which is impressive for a low-income country (4), Sri Lanka is still under-developed in emergency medical and pre-hospital care.
Though Sri Lanka faces natural disasters as well as mass casualty incidents frequently, it does not have a well-established system to respond to those disasters such as droughts, floods, tsunami, landslides, cyclones, tidal waves, etc. To respond immediately, standard pre-hospital and emergency medical services with special preparedness in disaster response are necessary. Traumatic injuries are ranked as the first leading cause for hospital admissions over the last ten years in Sri Lanka (5). It shows the need for emergency and retrieval medical services with good quality standards. Therefore, the objective of this study was to describe available facilities and equipment in ambulances to provide essential emergency care that transport patients to the National Hospital of Sri Lanka (NHSL).

\section{Methods}

A hospital-based, cross-sectional study was carried out from August to October 2008 at the NHSL. Every ambulance arriving at the accident service, outpatient department or emergency treatment unit of the hospital during the time of data collection was selected for the study. Any ambulance without an emergency patient was excluded. Once an ambulance was selected for the study, a sticker was pasted by the research team on the front screen of the ambulance, so that it was not repeatedly included in the sample, each time it arrived at the NHSL during the study period.

We could not find any studies done in Sri Lanka related to ambulances or emergency medical services at the planning stage and therefore, $50 \%$ was considered as the proportion of facility availability when calculating the sample size. Further, with $95 \%$ confidence level and an acceptable margin of error of 0.05 , the required sample of ambulances was 384 . After adding $5 \%$ as non-response from ambulance drivers, the number of ambulances to be enrolled was 403 . To obtain this sample, a probability sampling method was not applied due to practical difficulties, however data collectors adhered to a pre-determined method of data collection (six hour shifts each day). There were eight trained data collectors, working in four groups. They were trained using a fully-equipped ALS ambulance of the fire brigade and re-trained by a consultant anaesthetist at intensive care units/theatre setting to enable them to go through the checklist confidently. Two groups were allocated to accident service and the other two to the out-patient department and 
emergency treatment unit on a rotational basis on every other day.

A checklist for ambulance facilities and equipment was prepared to assess the available facilities and equipment required to treat patients in emergency situations in ambulances. It was prepared according to the standards of facilities that should be available in BLS and ALS ambulances in a developed country (1) and revised by a consultant anaesthetist in Sri Lanka. As there were no designated BLS and ALS ambulances in the Sri Lankan set up, all the facilities and equipment that should be available in such ambulances were included in a single checklist. Ethics clearance was obtained from the Ethics Review Committee of Faculty of Medicine, University of Kelaniya, and permission to carry on the study at NHSL was obtained from the director, NHSL.

The data were analysed using Statistical Package for Social Sciences (SPSS) version 16.0. Frequencies and proportions were calculated.

\section{Results}

Facilities available in 409 ambulances that arrived at the NHSL were observed. Among these ambulances, the majority ( $n=376,91.9 \%$ ) were from government hospitals, while military hospital ambulances comprised the second largest number $(n=12,2.9 \%)$. Of those ambulances from the government hospitals, the majority were from teaching hospitals, general hospitals and base hospitals ( $n=304,80.9 \%$, , while others were from district hospitals, peripheral units, estate and rural hospitals, central dispensaries and maternity homes $(n=72,19.1 \%$,$) . The largest number of ambulances$ were from hospitals located in the district of Colombo $(n=145,35.5 \%)$, while $63.2 \%(n=258)$ of ambulances in total were from the Western Province. The least number of ambulances were from Badulla, Ampara and Hambantota Districts. No ambulance arrived from the districts of Mullaitivu, Kilinochchi, Mannar and Monaragala. There were only 19 (4.6\%) communitybased ambulances, which brought patients directly from home/ field.

The ventilation and airway equipment that should be available in BLS ambulances, oxygen delivery equipment such as oxygen cylinders $(68.7 \%)$, flow regulators $(62.3 \%)$, oxygen tubes $(63.1 \%)$, and adult oxygen masks $(62.8 \%)$ were available in most of the ambulances. Equipment such as oro-pharyngeal airways $(4.9 \%)$, paediatric oxygen masks $(8.8 \%)$ and bulb syringe $(0.2 \%)$ were available in a few ambulances. Of all types of ventilation and airway equipment that should be available in ALS ambulances, only less than $1 \%$ was available. (Table 1 ).

\section{Table 1. Ventilation and air way equipment available for basic life support (BLS) and advanced life support (ALS) ambulances $(n=409)$}

\begin{tabular}{lr}
\hline Ventilation and air way equipment & No. $(\%)$ \\
\hline BLS ambulance: & \\
- Portable suction unit & $107(26.2)$ \\
- Suction unit & $156(38.1)$ \\
- Suction catheters & $159(38.9)$ \\
- Bulb syringe & $1(0.2)$ \\
- Oxygen cylinders & $281(68.7)$ \\
- Oxygen flow regulators & $255(62.3)$ \\
- Oxygen tubes & $258(63.1)$ \\
- Portable oxygen bottles & $86(21.0)$ \\
- Nasal cannula & $147(35.9)$ \\
- Oxygen masks - Adult & $257(62.8)$ \\
- Oxygen masks - Paediatric & $36(8.8)$ \\
- Bag valve mask resuscitators & $46(11.2)$ \\
- Oropharyngeal air ways & $20(4.9)$ \\
\hline
\end{tabular}

ALS ambulance:

- Endotracheal tubes - Adult $3(0.7)$

- Endotracheal tubes - Paediatric $1(0.2)$

- Laryngoscope and blades straight $7(0.5)$

- Laryngoscope and blades curved 6(0.5)

- Magill forceps - Adult $2(0.5)$

- Magill forceps - Paediatric $1(0.2)$

- Portable ventilator $3(0.7)$

Patient assessment equipment was available only in nearly $2 \%$ of all ambulances, such as blood pressure cuffs $(1.7 \%)$, stethoscopes $(1.7 \%)$, cardiac monitors $(1.5 \%)$ and defibrillators $(1.5 \%)$. Out of all the ambulances, pen-light and ECG electrodes were available in $19.6 \%$ and $13.9 \%$, respectively (Table 2). Splinting equipment and dressing material except bandages $(23 \%)$ and gauze $(6.4 \%)$ were available in 
less than 5\% of all ambulances (Table 3). Body substance isolation equipment, containers for disposal of waste and biohazards were available in $29.6 \%$ of

\section{Table 2. Patient assessment equipment available for a basic life support (BLS) ambulance $(n=409)$}

\begin{tabular}{lr}
\hline Patient assessment equipment & No. $(\%)$ \\
\hline Blood pressure cuffs - Adult & $7(1.7)$ \\
Blood pressure cuffs - Child & $2(0.5)$ \\
Blood pressure cuffs - Infant & $1(0.2)$ \\
Stethoscope & $7(1.7)$ \\
Pen light & $80(19.6)$ \\
Blood pressure apparatus & $9(2.2)$ \\
Portable trauma kit & $9(2.2)$ \\
Cardiac monitor & $6(1.5)$ \\
Defibrillator & $6(1.5)$ \\
ECG electrodes & $57(13.9)$ \\
Pulse oximeter & $8(2.0)$ \\
Electronic blood glucose monitoring device & $9(2.2)$
\end{tabular}

Table 3. Splinting equipment and dressing material available for a basic life support (BLS) ambulance $(n=409)$

\begin{tabular}{lr}
\hline Splinting equipment and dressing material & No. $(\%)$ \\
\hline Lower extremity traction splint & $4(1.0)$ \\
Upper and lower traction splint & $6(1.5)$ \\
Triangular bandages & $14(3.4)$ \\
Long board & $18(4.4)$ \\
Scoop & $12(2.9)$ \\
Vacuum mattress & $10(2.4)$ \\
Kendrick extrication device & $12(2.9)$ \\
Pediatric spine board & $7(1.7)$ \\
Head immobilization equipment - Adult & $8(2.0)$ \\
Head immobilization equipment - Paediatric & $6(1.5)$ \\
Cervical spine immobilization equipment & \\
- Adult & $4(1.0)$ \\
Cervical spine immobilization equipment & \\
- Paediatric & $3(0.7)$ \\
Bandages & $94(23.0)$ \\
Multiple dressing/ gauze & $26(6.4)$ \\
Sterile burn sheets & $15(3.7)$ \\
Adhesive tapes & $13(3.2)$ \\
Kidney basins & $11(2.7)$ \\
\hline
\end{tabular}

ambulances while disposable gloves and surgical masks were available in $24.2 \%$ and $15.9 \%$, respectively (Table 4). As for the safety and accessory equipment, most ambulances were equipped with fire extinguishers (75.3\%), protective restraints for patients and crew $(95.6 \%)$, wheeled stretcher $(95.4 \%)$, spare tire $(91.7 \%)$, tool kit $(79.2 \%)$, warning siren $(94.4 \%)$ and warning reflectors $(65.3 \%)$ (Table 5). Every type of obstetrical supplies was available only in less than $1 \%$ of ambulances (Table 6). All intravenous (IV) accessories except the IV hook $(89.2 \%)$ were available in less than $5 \%$ of ambulances and every type of drug delivery devices were in less than $2 \%$ of ambulances (Table 7).

Table 4. Body substance isolation equipment available for a basic life support (BLS) ambulance $(n=409)$

\begin{tabular}{lr}
\hline Body substance isolation equipment & No. $(\%)$ \\
\hline Disposable gloves & $99(24.2)$ \\
Goggles/protective eye wear & $9(2.2)$ \\
Surgical masks & $65(15.9)$ \\
Containers for disposal of waste & \\
and biohazards & $121(29.6)$ \\
Containers for infectious waste & $5(1.2)$ \\
Containers for sharps & $3(0.7)$ \\
Helmets/safety caps & $3(0.7)$ \\
Safety boots & $3(0.7)$ \\
\hline
\end{tabular}

\section{Table 5. Safety and accessory equipment available for a basic life support (BLS) ambulance $(n=409)$}

\begin{tabular}{lr}
\hline Safety and accessory equipment & No. $(\%)$ \\
\hline ABC fire extinguishers & $308(75.3)$ \\
Child safety seat & $2(0.5)$ \\
Protective restraints for patients/crew & $391(95.6)$ \\
Wheeled stretcher & $390(95.4)$ \\
Triage tags & $7(1.7)$ \\
Spare tire & $375(91.7)$ \\
Hack saw & $3(0.7)$ \\
Portable hand light & $230(56.2)$ \\
Tool kit & $324(79.2)$ \\
Warning siren & $386(94.4)$ \\
Warning reflectors & $267(65.3)$ \\
\hline
\end{tabular}


Table 6. Obstetrical supplies available for a basic life support (BLS) ambulance $(n=409)$

\begin{tabular}{lc}
\hline Obstetrical supplies & No. $(\%)$ \\
\hline Sterile obstetric kit & $3(0.7)$ \\
Towels & $3(0.7)$ \\
$4 * 4$ dressings & $2(0.5)$ \\
Cord clamps & $1(0.2)$ \\
Scissors & $3(0.7)$ \\
Bulb syringe & $2(0.5)$ \\
Sterile gloves & $3(0.7)$ \\
Thermal blanket & $1(0.2)$ \\
Neonate stocking cap & $1(0.2)$ \\
\hline
\end{tabular}

Table 7. Intravenous equipment and drug delivery devices available for an advanced life support (ALS) ambulance $(n=409)$

\begin{tabular}{lr}
\hline $\begin{array}{l}\text { Intravenous equipment and drug } \\
\text { delivery devices }\end{array}$ & No. $(\%)$ \\
\hline Normal saline & $14(3.4)$ \\
N/2 saline & $11(2.7)$ \\
N/5 saline & $7(1.7)$ \\
Lactated ringers solution & $4(1.0)$ \\
IV cannula neonate (yellow) & $5(1.2)$ \\
IV cannula infant and child (blue) & $9(2.2)$ \\
IV cannula adult (pink) & $8(2.0)$ \\
IV cannula adult (green) & $9(2.2)$ \\
IV cannula adult (white) & $10(2.4)$ \\
IV cannula adult (ash) & $6(1.5)$ \\
IV administration set & $20(4.9)$ \\
IV hook & $365(89.2)$ \\
Intravenous arm board - Adult & $8(2.0)$ \\
Intravenous arm board - Paediatric & $2(0.5)$ \\
\hline Drug delivery devices & \\
\hline Syringes 1cc & No. $(\%)$ \\
Syringes 2.5cc/ 3cc & $6(1.5)$ \\
Syringes 5cc & $6(1.5)$ \\
Needles 22G & $7(1.7)$ \\
Needles 18G & $6(1.5)$ \\
Paediatric drug dosage calculation equipment & $2(0.5)$ \\
\hline & $7(1.7)$ \\
& $7(1.7)$ \\
& \\
\hline
\end{tabular}

\section{Discussion}

The study focused on assessing the availability of standard facilities and equipment in ambulances. The majority of ambulances were equipped with only very basic and minimum facilities, such as wheeled stretcher and warning siren that have no direct effect on patient management. Only a very few were equipped with facilities that should be available in ALS ambulances, while the availability of some of these items was less than $1 \%$. To conduct this study, we selected the NHSL because it is the premier tertiary care hospital in Sri Lanka that receives emergency patients from all over the country. Therefore, it had a higher chance of obtaining a sample of ambulances from all over the country, which enhances the generalizability of results.

The studies done in other countries have revealed that most of the ambulances are equipped with necessary equipment at least on basic life support, though some equipment shortages are mentioned (3, 6). An Iranian study found that more than $66 \%$ of the ambulances had all equipment related to airway/ ventilation management (7). According to our study, necessary paediatric equipment was available only in less than $2.5 \%$ of ambulances. In concurrence, studies conducted in other settings (7-8) have reported that the availability of paediatric equipment was poor compared to adult facilities and equipment, but not as less as in our study. A study conducted in another developing country (9) reported that $99 \%$ of the ambulances had facilities to measure blood pressure and pulse rate, however less than $1 \%$ had automated monitoring devices to measure blood pressure, heart rate and pulse oximeter.

The data collection of this study was done in late 2008. However, there are no published studies to date that have assessed the level of facilities available in ambulances in Sri Lanka. According to our study, 4.6\% were community-based ambulances while all others were used for retrieval services. Having traumatic injuries as the leading cause of hospital admissions in Sri Lanka, a well-developed community ambulance service is mandatory. Recently, a community-based emergency ambulance service was launched in 2016 covering Western and Southern Provinces. This free service transports 130-135 patients daily. This service is not managed under the Ministry of Health. The facilities available in these ambulances are not documented. However, ambulance services are still functioning as a method of patient transport and retrieval 
service, rather than as an emergency medical service in Sri Lanka.

A review recommended the necessity of an accreditation system to meet international standards for proper emergency patient transportation services in South Asia (10). This is highly relevant to Sri Lanka, as it faces natural disasters almost every year. To be active without delay, standard pre-hospital and emergency medical services with special preparedness in disaster response are necessary. At present, there are no well-developed standards, guidelines or rules regarding emergency medical services in Sri Lanka. During the time of this study, there had been mass casualty incidents frequently due to the internal civil conflict in the country.

\section{Conclusions and Recommendations}

In conclusion, the standard facilities that should be available in both BLS and ALS ambulances were poor. Most of the equipment availability in ALS ambulances was extremely poor while the availability of equipment and facilities that should be available in BLS ambulances too was not up to standards. It is clear that national guidelines should be drawn to ensure safety and quality of the emergency care provided in ambulances, by assessing the facilities and equipment available for patient care. We recommend that there should be better policies for emergency care including standard checklists for all patient transport ambulances.

\section{Public health implications}

The equipment and facilities that should be available in ambulances for saving life of the patients were not up to the standard.

\section{Author Declarations}

Competing interests: The authors declare that they have no competing interests.

Ethics approval and consent to participate: Administrative clearance was obtained from the Director of the NHSL. Informed written consent was obtained from the ambulance drivers. Ethical clearance was taken from Ethics Review Committee of the Faculty of Medicine, University of Kelaniya (ERC number P52/07/2008).

Funding: Self-funded
Author contribution: Both authors (GN \& CA) have contributed equally to the design of the study, and analysed and interpreted the data. GN was responsible for the implementation of study and a major contributor in writing the manuscript. Both read and approved the final manuscript.

\section{References}

1. Department of Public Health \& Environment. Health facilities and emergency medical and trauma care system. Rules pertaining to emergency medical services, 6CCR 1015-3, Education and Certification (Chapter 1). Colorado, 2013.

Available from: https://www.colorado.gov/pacific/ cdphe/hfems

2. Committee on Trauma. Equipment for ambulances. American College of Surgeons.

Available from: https://www.facs.org/ /media/files/ quality\%20programs/trauma/.../ambulance.ashx

3. Marozas R, Rimdeika R, Jasinskas N, Vaitkaitiene E, Vaitkaitis D. The ability of Lithuanian ambulance services to provide first medical aid in trauma cases. Medicina (Kaunas) 2007; 43(6): 463-471.

4. Abeykoon P. The implications of technology change for health care delivery in Sri Lanka. Geneva: World Health Organization, 2003.

Available from: http://citeseerx.ist.psu.edu/viewdoc/ download?doi=10.1.1.604.1118\&rep=rep1\&type=pdf

5. Medical Statistical Unit. Annual Health Bulletin 2015, Sri Lanka. Colombo: Ministry of Health, 2015.

6. Vatankhah S, Kazamneghad E, Pourshaikhian M. A comparative study of road ambulances equipment based on the national standards in Guilan Province. International Journal of Health System and Disaster Management 2013; 1(1): 1-6.

7. Roberts K, Jewkes F, Whalley H, Hopkins D, Porter K. A review of emergency equipment carried and procedures performed by UK front line paramedics on paediatric patients. Emergency Medical Journal 2005; 22: $572-576$

8. Gaffney P \& Johnson G. Paediatric prehospital care: postal survey of paramedic training managers. Archives of Disease in Childhood 2001; 84(1): 82-83.

9. Crandon IW, Harding HE, Williams EW, Cawich SO. Inter-hospital transfer of trauma patients in a developing country: a prospective descriptive study. International Journal of Surgery 2008; 6: 387-391.

10. Choudry UK, Mahwish A, Areeba N. Emergency patient transportation in South Asia - call for a proper pre-hospital care system. Journal of Surgery \& Emergency Medicine 2017; 1(1:4): 1-3. 